\title{
Cognitive science applied to reduce network operation margins
}

\author{
Luis David Notivol Calleja ${ }^{(1)}$, Salvatore Spadaro ${ }^{(1)}$, Jordi Perelló( ${ }^{(1)}$ and Gabriel Junyent ${ }^{(1)}$
}

(1) Universitat Politècnica de Catalunya (UPC) - Barcelona Tech, Jordi Girona 1-3, 08034 Barcelona,

Spain, e-mail: \{david.notivol, spadaro, junyent\}@tsc.upc.edu, perello@ac.upc.edu

\begin{abstract}
In an increasingly competitive market environment with smaller product offer differentiation, a continuous maximization of efficiency, while guarantying the quality of the provided services, remains a main objective for any telecom operator. In this work, we address the reduction of the operational costs of the optical transport network as one of the possible fields of action to achieve this aim. We propose to apply cognitive science for reducing these costs, specifically by reducing operation margins. We base our work on the Case Based Reasoning ( $C B R$ ) technique by proposing several new schemes to reduce the operation margins established during the design and commissioning phases of the optical links power budgets. From the obtained results, we find that our cognitive proposal provides a feasible solution allowing significant savings on transmitted power that can reach a $49 \%$. We show that there is a certain dependency on network conditions, achieving higher efficiency in low loaded networks where improvements can raise up to $53 \%$.
\end{abstract}

\section{INTRODUCTION}

In the current fierce telecom market, a network operator will try to maximize commercial benefits in the trade-off between investments on the network (CAPital EXpenditures, CAPEX) and operational costs (OPerational EXpenditures, OPEX). A potential line of work to get savings, and therefore efficiency, can be to reduce the operation margins applied in the power budgets of the optical transport network links, configured during the design phase and used throughout the exploitation and operation of the network.

The operation margins are dimensioned and established during the design phase of the optical network to take into account unforeseen link degradations, due to the ageing of the network infrastructure among other reasons. Traditional techniques to calculate and set these margins are based on commercial tools, and they are adjusted during the commissioning phase, only once and at the beginning. They normally have a fixed value. Several criteria can be considered when setting their value, depending on the infrastructure provider or network operator policies. They are dimensioned taking into account the end-of-life (EOL) of the optical network components (fiber, connectors, nodes).

Our proposal makes use of the cognitive science to explore a solution improving the application of these margins. The solution proposed consists in considering the possibility that the operation margins will not have permanent but variable values; besides, it leverages on the idea that it might not be necessary to apply the worst-case value from the very beginning of 
optical network exploitation. Thus, the operation margins might be variable and adapt to the network conditions, e.g., by setting low values during the first steps of the network lifetime and get increased when optical infrastructure will age and degrade, in order to guarantee the feasibility of the end-to-end communications.

To reach this objective, we propose the application of the cognitive science [1][2]. The cognitive process means to be aware of the external environment, to reflect on the internal and external knowledge, to think based on this knowledge, to take a decision and to act or to plan, while learning constantly from the experience [3]. Previous works about the application of cognitive techniques to an optical network have been presented in a real-time context [4], that is, to assist in the online establishment of the lightpaths. We propose in this work a different line of action: the application of the cognitive science to take into account the longterm ageing process of the components when setting the optical power margins of the lightpaths to be established. Our main objective will be to reduce the operation margins and thus the OPEX of the telco operator with cognitive solutions. In particular, the technique chosen for fulfilling this objective is Case Base Reasoning (CBR).

The problem of reducing network margins is addressed in [1], where the different network margins are discussed, as well as the corresponding strategies to reduce them. Some of them consist on adapting the symbol rate, channel spacing and modulation format. The introduction of flexible transceivers is also considered to address the problem. More recently, the work on network margins categorization proposed in [1] has been further developed in [5] with a review of their characteristics and an explanation of the strategies and technologies to exploit them and translate them into additional capacity. The authors highlight the challenge of implementing them in real networks: margins can be reduced through careful network planning, using flexible equipment (for example, transponders and optical and electronic switching fabrics) and monitoring techniques [5]. There are other works that propose to apply cognitive techniques to optical networks to build a cognitive Quality of Transmission (QoT) estimator for classifying lightpaths into high or low categories, which is much faster for on-line operation than existing approaches [4]. This estimator computes the associated Q-factor values of the lightpaths and assesses their QoT before their establishment, based on the CBR technique.

As highlighted above, one of the network operators needs is to reduce OPEX as much as possible. Moreover, with the introduction of flexible transponders and higher order modulation formats, capacity can considerably increase but with a significant reduction in reach. A strategy to overcome this limitation can be to reduce margins in network [1]. During the design phase of the optical network, one of these network margins, the System Margin, introduced to cope with unforeseen degradations, is estimated and used to calculate the link power budget. The System Margin is one of the components involved in the power link budget calculation, where transmission power is worked out and adjusted. Once this parameter is set and fixed, network operators and infrastructure suppliers should use a fixed constant value all through the entire lifetime of the optical transport network; furthermore, it uses to be the same value for any network conditions in any situation. Once we have stated the addressed problem, we propose a cognitive approach to solve it. 
The remainder of this paper is structured as follows. In section 2, the employed CBR cognitive technique is briefly explained. In section 3 , we present the development of the work done. In section 4, we present the obtained numerical results. Finally, section 5 draws up some conclusions.

\section{CASE BASED REASONING TECHNIQUE}

Machine learning deals with the study of algorithms which improve their performance for a specific task based on experience, and adapt automatically to situations without a previous and explicit programming of the system [6].

In this work, we have particularly focused on the Lazy Learning techniques, in the Supervised Learning, Non-Linear Decision category [6][7]. This machine learning branch waits for starting learning, and then for the generalization, until a new attempt arrives to the system. An advantage of this strategy is to solve several different problems simultaneously. Moreover, it can more easily manage the problem domain. This characteristic may result very useful in the context of our work, when a new traffic request arrives to the optical network, whose state at that moment has to be considered.

Inside the Lazy Learning strategies, we have selected CBR [5], an advanced technique of Instance Based Learning (IBL) [5], due to several reasons: it is applicable to build structural and complex descriptions of the optical network. Also, CBR techniques provide local approximations to the target function: we want to exploit this characteristic in our framework because we want to obtain the best solution in a particular moment in the optical network, and not a general solution to the problem. CBR is based on the previous experience to solve new situations: similar problems have similar solutions and situations often repeat. This technique approaches to the human thinking and reasoning: a new problem is tried to be solved by finding similar past experiences stored in the memory and reusing them by adapting and applying total or partially the previous solution to the new situation. CBR has a relatively fast training phase, as a Lazy Learning algorithm, and can learn complex functions in a relatively simple way (i.e., a solution can be quickly proposed and the domain under analysis does not need to be fully understood). Additionally, it is a technique that requires less maintenance effort compared to alternative cognition techniques and can adapt to changes in the environment. Its main drawback, however, is the storage capacity or memory needed to store past experiences; CBR can also be revealed as low to solve specific problems because learning is done when a new query arrives. In view of this, our proposal is to perform learning offline and not in a real-time context as previous works using CBR proposed [4]. Other disadvantages are that best cases are not always selected and applied and memory can contain irrelevant information.

CBR is based on a Nearest Neighbor classifier. It is a methodology based on a 4-phase cycle: Retrieve, Reuse, Revise and Retain. The initial Retrieve phase consists in finding similar past problems, modeled as "cases" with several attributes stored in memory, that is, in a database called Knowledge Base (KB). The concept of similarity is a key point because it conditions the utility and reusability of such cases. Particularly, it is modeled and calculated as the distance between the query point and the similar past cases. Then, retrieval is based on the nearest neighbor algorithm, usually using the $\mathrm{k}-\mathrm{NN}$ variant (considering $\mathrm{k}$ neighbors). The second phase, Reuse, consists in proposing solutions to the current situation based on the retrieved 
cases, by reusing their past solutions. To this purpose, we propose two new schemes, described in the following section. The third phase, Revise, consists in adapting and repairing the proposed solution; the substitution of some parts of the retrieved solution is commonly the simplest form of adaptation. The Revise phase is also responsible for verifying and correcting the solution. Finally, the fourth phase, Retain, consists in storing the new case and its solution in the KB (i.e., learning it) for upcoming lightpath allocation events.

\section{PROPOSED APPROACH}

The target of this work is to apply and verify the utility of the CBR cognitive technique in the optimization of the optical links power budget. Our cognition-based proposal will provide a new transmission power value, which will allow setting the System Margin parameter for an end-to-end path.

To this end, we have assumed a National 9-node long-haul optical network (Figure 1). In particular, we focus on four representative end-to-end paths across the network, composed of several optical links and spans with intermediate ROADM/WSS nodes considered as passthrough Optical Amplifier nodes. Each path has been characterized by the following attributes: length, number of in-line amplifiers, number of simultaneous wavelengths allocated and minimal spectral distance among them, non-linear impairments and net link losses, modeled by the accumulated losses in the fiber, the connectors and the splices. Over each path, traffic requests over time have also been considered, modeled by requested throughput, required quality for the connectivity service, characterized with the BER, and the required sensibility of the receiver for each request. And, finally, the design parameter, i.e., the System Margin, set in the end-to-end path. These attributes define each CBR case, whose output is the optical power value to be emitted.

As already introduced, the System Margin is the security margin in the power budget applied to cope with unforeseen degrading effects. A fixed conservative value is typically set during the design phase, which takes into account the end of life of components. This value is adjusted during the commissioning phase of the network and applied during the operation of the optical network. We propose to not use this value from the beginning of the exploitation of the network, but instead to use a lower and flexible value, guaranteeing in any case the required transmission quality over the path. Specifically, we aim to use the cognitive CBR technique to determine this value, as a function of the state and ageing of the different components of the optical network.

We have applied the following basic power balance equation to model the end-to-end path as point-to-point link between two end nodes:

$$
\mathrm{Ptx} \geq \mathrm{Srx}+\mathrm{CL}+\mathrm{NLI}+\mathrm{M}
$$

Specifically, Srx is the sensitivity of the optical receiver, that is, the minimal optical power to be received, carried out for every new demand. It has been established based on the Quantum Limit of the receiver [8] [9] and adapted to the typical values used by a commercial network operator, trying to be agnostic of the infrastructure provider. The allowed values range varies between $-24 \mathrm{dBm}$ and $-12.5 \mathrm{dBm}$. CL models the channel net losses, including fiber, connectors and splices, which will increase as time passes due to components degradation. We have 
established the attenuation range between $12 \mathrm{~dB}$ and $24 \mathrm{~dB}$. NLI includes the non-linear impairments and the power penalties in the DWDM long-haul amplified and non-regenerated systems. Among them, we have selected the phenomena more likely to present and affect a commercial DWDM network such as SRS (Stimulated Raman Scattering), SBS (Stimulated Brillouin Scattering), SPM (Self-phase Modulation), XPM (Cross-phase Modulation), FWM (Four-wave Mixing), PDL (Polarization Dependent Loss), PMD (Polarization Mode Dispersion), Crosstalk and Power Varying. In the literature, different criteria and typical value ranges for them can be found [8] [9] [10] [11] [12] [13] [14]. Allowed NLI range considered in our work varies then from $0.02 \mathrm{~dB}$ and $5 \mathrm{~dB}$. Moreover, if the path transports traffic requests above 40 $\mathrm{Gb} / \mathrm{s}$, we have added additional $1.5 \mathrm{~dB}$ due to power penalties depending on the number of spans and crossed high-power amplifiers, Hybrid Transmission Penalties (around $1.2 \mathrm{~dB}$ ) and the crossed ROADM (the path passed through the node). $M$ represents the System Margin, also called Security Margin or Operator Margin. This value both in the literature and in an optical commercial network can vary considerably: between $5 \mathrm{~dB}$ and $10 \mathrm{~dB}$ including power penalties [9], between 3-6 dB [10], between 3-4.8 dB [12], between 4 and $6 \mathrm{~dB}$, including Power Penalties [8] and between 0 and $9 \mathrm{~dB}$ [11]. The considered range for our work will fluctuate between $1 \mathrm{~dB}$ and $4 \mathrm{~dB}$ to incorporate the unforeseen degradations and the aging effects. Regarding the transmission power in the Optical Transmission Units boards, we have set a grid of discrete allowed values typically used in commercial networks between $[7.5,-2.5]$ $\mathrm{dBm}$, with a step of $0.5 \mathrm{dBm}$. Finally, we have chosen four different paths between network nodes: paths from one to four links, with physical lengths of 110, 350, 460, $785 \mathrm{Km}$, respectively, with variable span lengths in the links (in-line amplifiers not being installed following a constant length).

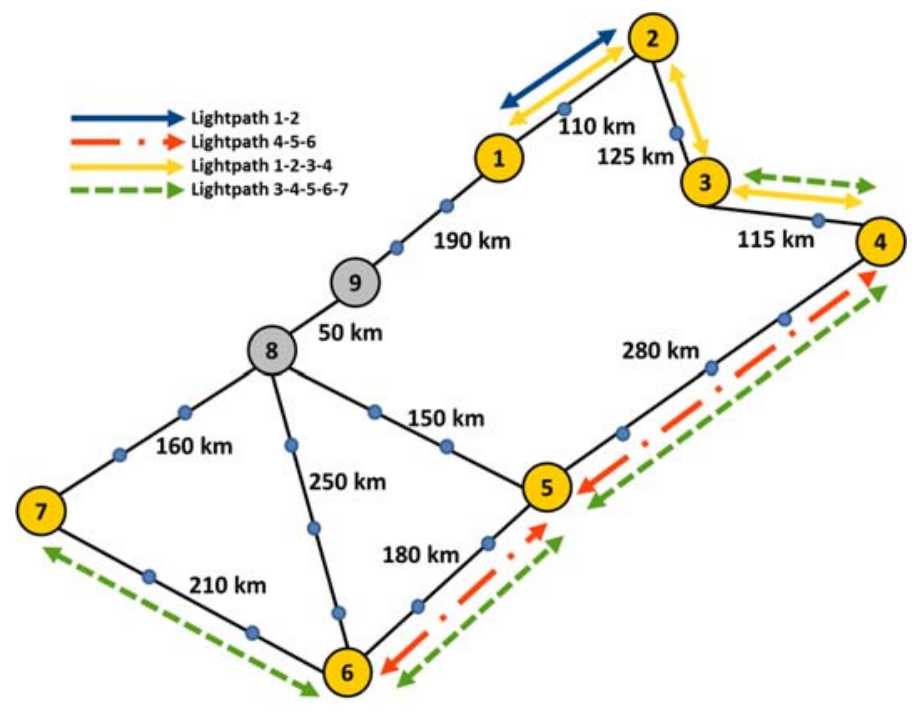

Figure 1. Network topology used

In the power budget (in)equation (1) some of the parameters ( $C L, N L I)$ are random variables with values between the indicated ranges. In real network scenarios, however, quickly computing their exact value is a complex task and not always possible (it would also require a complex monitoring system). This is because there is some uncertainty, unknown beforehand. 
These uncertain values of the ageing component and non-linear impairments have been modeled by $\mathrm{CL}$ and NLI parameters, which are taken into account by CBR when evaluating performance. Then, CBR is able to learn from the data how ageing is affecting to the required transmission power, without being forced to know accurately the random values in order to propose a successful transmission power. This is the advantage of using CBR: to use past situations to assign a transmission power value (thus, a System Margin value) to the new connections, overcoming the complexity above indicated.

We assume that the Knowledge Base stores 300 cases or past experiences, collecting a set of previous observations in the network containing, among other attributes, the transmission power (Ptx) and the System Margin (M) used.

In our approach, the KB is a database containing previous successful LPs. We assume that the $K B$ is populated offline with successful test lightpath establishments performed by the network operator. It could be renewed offline every certain time window (e.g., per month or year), by performing appropriate measurements in new test LPs set up by the operator.

Therefore, in our study, the initial KB has been generated following an offline learning strategy, based on measurements performed by the operator at different (historical) moments by launching test LPs. Such tests, performed offline, can rely on real measurements. In this way, those successful LP are then stored in KB together with the real measurements of their attribute values, which can afterwards be used by CBR for the allocation of incoming LP requests during the network operation. Then, the KB would be updated in a time scale in which ageing effects might change. It does not necessarily mean that all new candidate cases to be included in the KB will involve higher ageing effects. In fact, KB cases are not a chronologically ordered list of LPs, in which the System Margin becomes higher and higher because the network is getting older and it is necessary to increase the System Margin. Conversely, they are historical precedent successful cases, which correspond to different traffic demand situations (modeled with BER, throughput and lambda parameters) in certain past network conditions modeled by NLI (non-linear impairments penalties) and CL parameters (modeling the net losses by several raisons as ageing or others). Thus, the KB does not store active LPs.

The rationale behind such an approach is that the whole network does not get older homogeneously. Hence, maintenance operations are not applied to all links and network equipment simultaneously. As a result, several network situations may be generated: the same LP can have some links that have been maintained and others that not. Then, network status and conditions for a same LP at different times can be distinct (in terms of ageing and nonlinear impairments). Collecting this diversity of network situations is the objective of the KB and an advantage of using CBR.

As explained above, the KB will only register successful past experiences allowing us to know the transmission power needed for guaranteeing the requested quality of the service (QoS), given some specific channel conditions and the eventually applied valid $M$ value, lower than the initial value established in the design $(M=4 \mathrm{~dB})$. 
To obtain the numerical results in the paper, the transmission power of the successful cases in the KB was computed using equation (1) and a System Margin lower than $4 \mathrm{dBs}$. The NLI and $C L$ values have been randomly generated as if they would have been real measurements. Thus, for every case, we have generated random values of the attributes among the indicated ranges and taken into account several conditions:

1) Ageing may probably affect in longer LPs (because more network components -fiber spans and nodes- are passed through).

2) NLI values are probably higher when more simultaneous connections are active and sharing some of the links.

3) NLI values are also likely to be higher when simultaneous connections sharing the links use closer wavelengths.

4) The System Margin may also be probably higher when ageing is higher.

Table 1 specifies the ranges of the attributes for generating the KB cases.

\begin{tabular}{|c|c|c|}
\hline Name & Minimum value & Maximum value \\
\hline Receiver sensitivity (Srx) & $-24 \mathrm{dBm}$ & $-12.5 \mathrm{dBm}$ \\
\hline Channel net losses (CL) & $12 \mathrm{~dB}$ & $24 \mathrm{~dB}$ \\
\hline $\begin{array}{c}\text { Non-linear impairments and power } \\
\text { penalties (NLI) }\end{array}$ & $0.02 \mathrm{~dB}$ & $\begin{array}{c}5 \mathrm{~dB} \\
(6.5 \mathrm{~dB}, \text { if request } \\
>40 \mathrm{~Gb} / \mathrm{s})\end{array}$ \\
\hline $\begin{array}{c}\text { System Margin or Operator } \\
\text { Margin (M) }\end{array}$ & $1 \mathrm{~dB}$ & $4 \mathrm{~dB}$ \\
\hline Bit error rate (BER) & $10^{-15}$ & $10^{-9}$ \\
\hline Throughput & $1 \mathrm{~Gb} / \mathrm{s}$ & $40 \mathrm{~Gb} / \mathrm{s}$ \\
\hline Number of simultaneous lambdas & 1 & 10 \\
\hline Transmission power (Ptx) & $-2.5 \mathrm{dBm}$ & $7.5 \mathrm{dBm}$ \\
\hline
\end{tabular}

Table 1. Parameters of the model to describe the scenario

New incoming lightpath allocation requests come with transmission power determined by the $\mathrm{M}$ parameter fixed and predetermined in the design phase. When a new request with a specific bandwidth and quality of service arrives, the transmission power used in precedent similar situations is revisited and the new optimized $M$ value is established, thus reducing its initial value set in the design.

CBR is applied during the network operation to every incoming LP request. This will allow us to select an optimized $M$ value, getting savings in the transmission power, which will extend the life of the transceiver, while reducing the maintenance costs for the telco operator. As indicated above, OPEX are the costs related to the network usage, such as energy consumption, network maintenance and fault reparation costs, as well as the underuse of the network devices arising from an undesirably short effective life [15].

We have generated $10^{4}$ new requests and applied CBR to obtain the new transmission power, adapted to each new arrival. We have established two indicators: "CBR\%" denoting the 
success rate of the cognitive methodology to propose a new transmission power; and "FitCases\%", that is used to quantify the \% of proposed solutions by CBR yielding a transmission power reduction, namely, suggesting a transmission power equal or lower than the pre-assigned power calculated with the initial $M=4 d B$ value. This last indicator is introduced to avoid solutions proposed by CBR that, although valid, suggest a larger transmission power than the pre-assigned power, hence inappropriate. We can observe that sometimes "FitCases\%" increases but the proposed transmission power remains in the same range as the one of the new case; then, even though the solution is correct, we cannot take direct advantage for the operational optimization of the network, since no power savings are obtained; only a solution proposing a transmission power in a lower grid range can result in effective power savings, thus, it is useful for operational purposes, by subtracting to the fixed System Margin of the new incoming case, the difference of both transmission power values. In this way, a reduction and an optimization of the System Margin are proposed and can also be applied in future similar situations, materializing savings in the operation margins.

The needed System Margin may increase over time because ageing effects will probably be more present over time. This situation is modeled in the KB with cases showing a higher $\mathrm{CL}$ parameter.

We can establish an upper threshold on the receiver side (based on received power or BER or OSNR) to decide increasing the System Margin as a preventive strategy. If a situation requiring a higher System Margin occurs, this increase will be applied to the affected existing connections by increasing its transmission power; in turn, this power increase will probably lead to a non-linear impairments higher value (e.g., crosstalk), having an effect on either the current connection and all the connections sharing the same links, which would involve in turn the increase of their System Margin, thus their transmission power. The application of the transmission power increase on the affected connection will be previously controlled by a function to automatically ensure that the optical power flatness is inside the ranges determined during deployment commissioning phase and below a given Power Unbalanced Threshold (i.e., $1.5 \mathrm{dBs}$ ). The required power increase will be upper limited by the maximum allowed transmission power channel in the system. If the required needed power exceeds the allowed limits, the connection should be rerouted to a protection path, although this has not been considered in this work.

The fundamental trade-off between the increase of the transmitted power to overcome noise impairments and limiting the power to avoid the impact of nonlinearities has to be taken into account as stated by equation (1). By increasing the transmitted power, the effect of nonlinearities progressively grows up inducing a stronger impairment on performance. The optical path parameter values set in the design phase define the limits of satisfactory operation of the link. Optical paths with values outside these limits may yield link performance that exceeds the required bit error ratio.

The potential loop existing in equation (1) in terms of increasing NLI and transmitted power is upper-limited, theoretically, by using the highest allowed transmission power (7.5 $\mathrm{dBm}$ ) established as an upper rule in the whole network. When setting the maximum value of the System Margin (4 dBs in our case) network designers are supposing that this value will be able 
to absorb the maximum non-linear impairments and ageing values that will be found in the network. New lightpaths requests established using as System Margin 4 dBs will be successfully established. In a real network, the maximum transmitted power is limited by the deployed technology and we have decided to set this maximum value to $+7.5 \mathrm{dBm}$.

The launch power is determined during network design between a MIN and MAX value. Equation (1) is upper-limited by a transmission power MAX value. And the NLI penalties are also limited by a MAX value, integrated in the power budget equation. If the NLI value increases, the minimum transmission power to satisfy equation (1) will also increase up to reaching the allowed transmission power MAX value. And the transmission power value cannot exceed the maximum allowed value (fixed during network design and set to $+7.5 \mathrm{dBm}$ in our implementation). Any commercial network is dimensioned following the values determined during the design phase. We have decided that the maximum NLI value taken into account for the maximum allowed transmission power is NLImax $=5 \mathrm{~dB}$ (or NLImax $=6.5 \mathrm{dBs}$ for requests above $40 \mathrm{~Gb} / \mathrm{s}$ ).

In a commercial network, there are additional monitoring security mechanisms warning that the Output Power Upper Threshold is about to be reached (or has been exceeded), and an alarm is generated to inform the user of this abnormality, ensuring the stability of the optical signal or preventing the fiber being burnt. And if it is finally required to launch a power higher that the maximum allowed one, the lightpath will not be established over that path.

The Retrieval phase function in CBR is normally based on the supervised learning Nearest Neighbor technique that, for any new incoming instance, seeks the most similar case stored in the KB. As a variant of the Nearest Neighbor technique, the k-NN algorithm looks for the $k$ nearest neighbors, trying to minimize the error risk due to spurious examples. In our implementation we have proposed a k-NN function for the Retrieval phase: it returns a list of cases, the $\mathrm{k}$ nearest neighbours of the testing instance. Similarity is calculated in terms of the distance between the new instance and the neighbors. To this goal, the standard Euclidean distance is typically used in the k-NN implementations. We have considered in our work a limit value for the $k$ parameter equal to $7(k=[1,7])$ and we have introduced in the distance calculation the $r$ parameter (Minkowski metric, see equation (3) later on), with a range $r=[1,3]$ ( $r=2$ is the Euclidean distance). In typical implementations, for discrete output variables $k-N N$ should return the Mode (value occuring most often); for continuous variables, k-NN should return the Mean.

Next, we take the k-NN function output for implementing the Reuse phase, where we propose two new algorithms to be used besides the commonly used Mean and the Distance-Weighted ones [6]. Specifically, we will combine the aforementioned k-NN function output cases according to four proposed schemes:

- MEAN: mean voting scheme

- DISTANCE-WEIGHT (DW): a dynamic weight scheme depending on the distance of each neighbor to the instance

- MIN: New proposal where the minimum value is selected

- FIXED-WEIGHT (FW): New proposal using a fixed weight scheme, according to the number of neighbors considered, with weights distributed in a decreasingly linear way 
The output of the each neighbor is the power transmitted in the observation modeled by that case successfully fulfilling the quality of service conditions in a similar previous situation. Each case is modeled by a vector of the attributes explained above, characterizing the observation:

$$
\left(x_{1}, x_{2}, x_{3}, \ldots x_{n}\right)
$$

And the distance between two cases or instances is calculated using the Minkowski metric as following:

$$
d(\text { case } x, \text { case } y)=\sqrt[r]{\sum_{i=1}^{n}\left|x_{i}-y_{i}\right|^{r}}
$$

The similarity is carried out in terms of the network conditions of the end-to-end path and the traffic request. Figure 2 illustrates the described process.

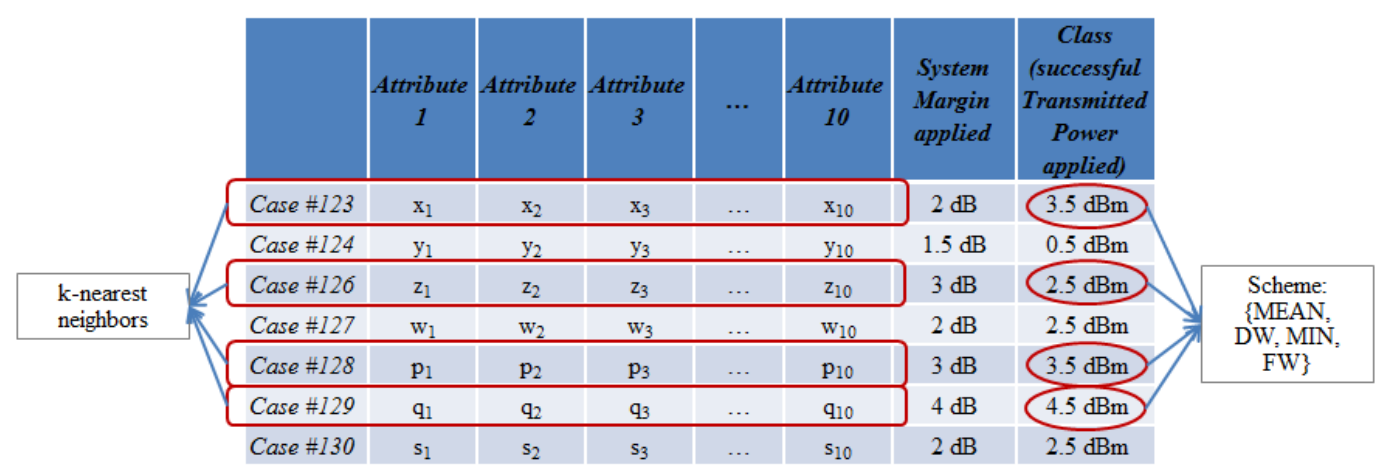

Figure 2. Example of the proposed CBR process.

In particular, the Retrieval phase uses $\mathrm{k}=4$ (and $\mathrm{r}=2$ ) selecting the 4 nearest neighbors in our $\mathrm{KB}$, that is, the 4 previous network situations more similar to the new incoming request. Each neighbor case used a successful transmission power. The Reuse phase can apply one of the 4 schemes to propose the new transmission power for the new incoming request. Table 2 depicts the transmission power values that each of the evaluated schemes would propose.

\begin{tabular}{|c|c|c|c|c|}
\hline & $\begin{array}{c}\text { MEAN } \\
\text { scheme }\end{array}$ & $\begin{array}{c}\text { MIN } \\
\text { scheme }\end{array}$ & $\begin{array}{c}\text { FW } \\
\text { scheme }\end{array}$ & $\begin{array}{c}\text { DW } \\
\text { scheme }\end{array}$ \\
\hline $\begin{array}{c}\text { Proposed } \\
\text { Transmission Power }\end{array}$ & $3.5 \mathrm{dBm}$ & $2.5 \mathrm{dBm}$ & $3.8 \mathrm{dBm}$ & $4.1 \mathrm{dBm}$ \\
\hline
\end{tabular}

Table 2. CBR transmission power proposed

The margin itself is not directly used when proposing a new transmission power, because it would lead us to an incorrect similarity computation, and this would prevent us to select those most similar neighbors. 
As explained before, LPs stored in the KB are not replaced with newer ones in the present work. However, even if an online replacement would have been considered, the transmission power of newly added LPs would not necessarily have to be higher, as ageing (and preventive maintenance) do not typically affect (is performed for) all links traversed by a LP homogeneously, but only one or some of them. Strategies to update the KB do not necessarily lead to new cases using higher transmission power, the KB is not a chronological ordered list of network situations cases; the KB tries to reflect as many heterogeneous network situations as possible, because network maintenance is not homogeneous (not applied to all the links and node elements at the same time). This is the reason why our strategy relies on an offline population of the KB database. As explained above, our approach aims to take into account the uncertainty in real network scenarios where CBR helps to manage. Thus, any CBR scheme proposing a lower transmission power can suppose an advantage.

The Revise phase simply encompasses the comparison between the proposed class (that is, the transmission power) by the Reuse phase and the correct one (the pre-assigned transmission power) supplied with the instance. If the proposed power satisfies the quality of service needed by the incoming request, then CBR\% firstly (monitoring the power budget) and then FitCases\% (monitoring the operational improvement) increase and we achieve power savings. On the other hand, if CBR\% indicator does not increase, it means that the proposed transmission power by CBR would lead to blocking of the new lightpath. In this case, the new incoming request uses the pre-assigned transmission power and no power savings is achieved. In our work, we can fix a threshold of $C B R \%=95 \%$ to determine if CBR fulfills our objective.

In our assumption, the Revise phase consists in a direct verification at the receiver. In particular, an unmodulated laser signal is sent and the corresponding power is monitored in order to detect whether the transmission power proposed by CBR is sufficient to balance the power losses. If the received power is not enough, a command is sent to the transmitter to increase the transmission power Ptx_default - Ptx_CBR dBs, being Ptx_default and Ptx_CBR the optical transmission power assuming the default System Margin and that proposed by CBR, respectively. Such test strategy can be triggered by a network controller (e.g., SDN-based). With this architecture in mind, CBR runs inside an SDN network controller module and the interaction with the physical devices is carried out through the south-bound interface, for example, by using the Openflow protocol. With this strategy, if the proposed solution is not valid, we directly increase the transmission power in order to guarantee the transmission.

Finally, in the Retain phase the instance should be stored in the memory structure as a new case if the policy allows it. This is not performed in this first work, thus being a non-learning classifier. However, several strategies for the Retain phase could be possible. For example, any new successfully established LP could be added in the KB. Or, alternatively, only those ones showing a significant difference with respect to the already included ones might be worthy to be added in the KB, so as to avoid the storage of redundant information. These strategies would certainly increase the KB size, and thus the CBR computation time and the required storage capacity. Hence, we could allow the addition of new LP only until reaching a certain threshold, limiting the maximum acceptable CBR computation time. Every time that an existing $L P$ in the KB was part of the nearest neighbors used by CBR to calculate the proposed power 
value, it could be marked by an incremental flag. Thus, candidate LPs to be kept in the KB would be those ones more frequently selected for CBR calculations. LPs less often (or never) selected would be removed from the KB. Another strategy could be to also add unsuccessful LPs in the KB (marking them with another flag, for example), that is, LPs whose received transmission power has not been enough to ensure their feasibility, as we can also learn from errors. All these possibilities could be contemplated in future works emanating from the present one.

\section{RESULTS AND DISCUSSION}

We propose two new algorithms on the policy used for CBR to provide a solution and analyzed some settings of the CBR methodology applied to different network conditions in terms of traffic load and that for several end-to-end paths.

As highlighted in section 3, we have considered 4 LPs of the network of Figure 1 for the whole study, which have been selected by following a representativeness criteria in terms of length and number of network components (number of fiber spans and number of network equipments, as online amplifiers and node elements), under the hypothesis that the ageing is probably affecting to the fiber and every node equipment. Following this criteria, we have selected the four LPs more representative of the network for the whole study. Other possible LPs in the network should be similar to the considered ones.

For every considered physical path and every statistical result (every combination of $\mathrm{K} / \mathrm{r} / \mathrm{scheme} /$ mean load variables) we have used $10^{4}$ offered lightpath requests.

Regarding the performance evaluation, the following attributes change in each request: BER, throughput, wavelength, number of simultaneous wavelengths in each link through a path, distance of the closest wavelength used in each link, NLI and CL. They have been randomly generated among the indicated ranges and considering several conditions (as explained in section 3). And every case has been generated in that way, for every case in the KB and for every new incoming traffic client request. Indeed, we do not have used the same values of NLI or net losses for all connections through the same path (the NLI generated value depends on the number of existing connections through a path).

\subsection{Selection of Knowledge Base size}

The first step in our work has been addressed to set the optimum size of our KB. We have selected a typical end-to-end path in the network, between nodes 1 through 4 (LP 1234), with 4 nodes and 3 links, a mean network load of $50 \%$ and values $k=3$ and $r=2$ in CBR. We have considered 3 possible KB sizes: 100, 300 and 500 cases. The analysis has been done with the four schemes previously presented for the Revise phase: MEAN, DW and our two proposed MIN and FW ones. The selection of the optimum KB size is decided looking at the success rate of the proposed transmission power by CBR (CBR\% indicator) combined with the amount of fit cases (FitCases\%), taking also into account the mean power savings achieved.

Figures 3 and 4 show the obtained results. Initially, KB size set to 500 cases seems the optimum one for any scheme. Nevertheless, looking at the volume of fit cases (those whose 
proposed transmission power is equal or less than the pre-assigned one), we observe no remarkable differences between the three possible studied KB sizes. As the value of FitCases\% is slightly higher for KB size 300 cases against KB size 500 (e.g., 98.38 vs. $98.29 \%$ for the MIN scheme, respectively), we set in a first approach the optimum KB size equals to 300 cases. In this first approach, it can be observed that the best results regarding the combination of the success rate of the proposed transmission power by $C B R$ and the number of fit cases is obtained with our proposed new algorithm MIN.

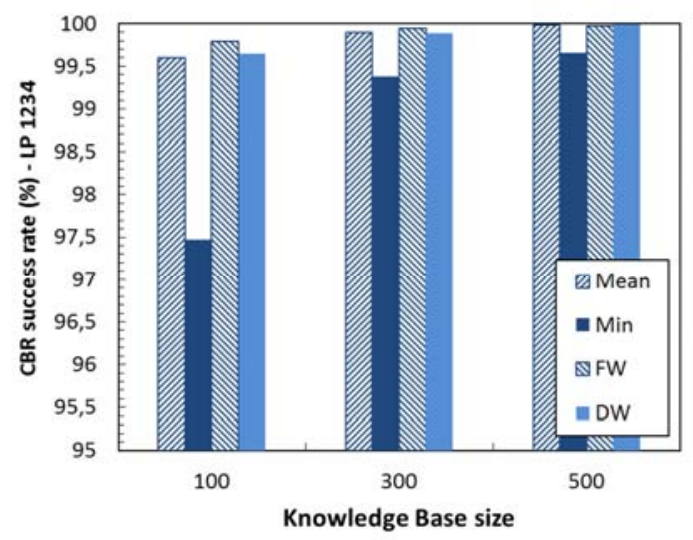

Figure 3. Evolution of CBR success rate for different KB sizes with network load $50 \%$ and path 1234

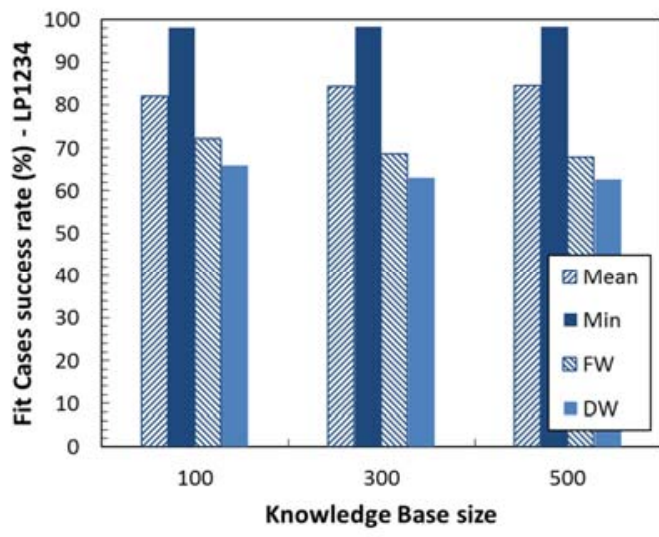

Figure 4. Evolution of FitCases\% for different KB sizes with network load 50\% for and path 1234

Therefore, the results obtained indicate that $\mathrm{CBR} \%$ rises when the $\mathrm{KB}$ size increases, for any of the four schemes, and in parallel, the power mean savings decrease. Taking into account the CBR\% threshold set above, 95\%, we have selected the optimum KB size as 300 cases, because this setting shall be also valid for longer paths with more links. By choosing KB size as 500 cases, first of all, we would obtain slightly poorer results regarding mean power savings. Moreover, we would increase the size of the KB (i.e., the storage/memory required) and the corresponding computation time (from 300 to 500 cases per lightpath, which considered for all possible end-to-end paths, it would be less efficient), without experiencing a significant impact in terms of success rate and power savings.

We have additionally performed the analysis of the impact of the number of links and the length of an end-to-end path on the CBR success rate and the mean power savings. Although not reported in this paper, the results indicate that for longer paths with more links, the CBR success rate slightly decreases, while the mean power savings increase, for any network load. The results for a KB size of 100 cases are also globally acceptable for path LP 1234 in terms of both CBR success rate and mean power savings. However, for longer paths with more links, CBR\% decreases and results are poorer than for KB size equal to 300 . For example, if we consider the path between nodes 3 and 7 (LP 34567), CBR\% becomes 93.91\% with KB size equal to 100 against $97.42 \%$ with KB size equal to 300 . On the other hand, as can be noted in Figure 3, the difference on CBR\% between $K B$ size 100 and 300 cases is more significant than between KB size 300 and 500 cases; as indicated above, we save computation time and 
capacity storage for all the lightpaths of the network when considering a lower KB size. As a conclusion, from now on, we set the optimum KB size to 300 cases.

\subsection{Selection of CBR parameters}

The next step in the analysis is to perform the fine tuning of the CBR parameters that will be used: $k$ (number of past cases more similar to the incoming request) and $r$ (determining the Minkowski metric used to calculate the degree of similarity). The outperforming scheme MIN is used. The end-to-end path considered is the LP 1234 with a mean network load still set as $50 \%$. We have evaluated the range [1-7] for the $k$ parameter and the range [1-3] for the $r$ parameter. Figures 3 and 4 show the obtained results.

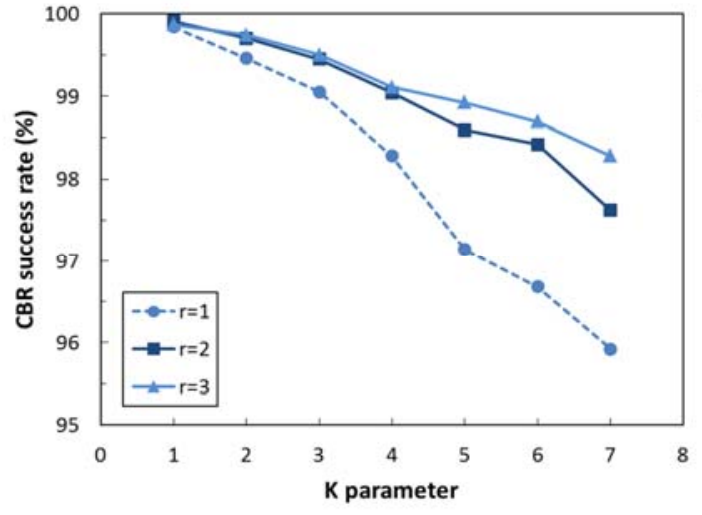

Figure 5. Evolution of CBR success rate with k, for path LP 1234, scheme MIN and mean network load $50 \%$

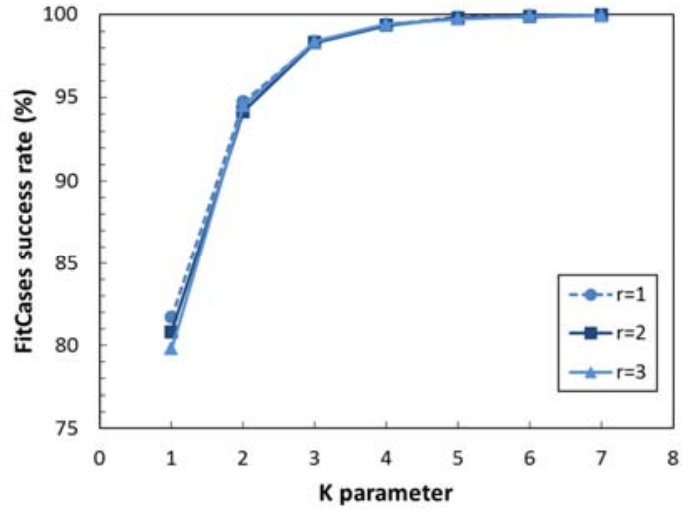

Figure 6. Evolution of FitCases\% with $\mathbf{k}$, for path LP 1234, scheme MIN and mean network load $\mathbf{5 0 \%}$

It can be observed that, for path LP 1234, applying our proposed scheme MIN with a mean network load of $50 \%$, the success rate of the cognitive technique decreases as $k$ increases. Simultaneously, the number of fit cases increases proportionally with the $k$ value. The best combination of $[k, r]$ parameters appears to be $k=5$ and $r=3$, if we consider the combination of the success rate of the proposed transmission power by CBR and the number of fit cases. The value $r=2$ can also be taken into account because the difference in CBR\% indicator between $r=2$ and $r=3$ is not significant, and the lower the $r$ value parameter is, the lower the computation time. For example, the CBR success rate becomes $98.59 \%$ and $98.92 \%$ with $r=2$ and $r=3$, respectively. In any case, we choose to discard $r=1$, which presents the lower results among the three possible values. For other mean network loads we have verified that results show the same trend, except for a mean network load of 100\%; in that case, the best result is obtained for $k=6$ and $r=3$. However there does not exist $a$, let us say, best $[k, r]$ combination common and valid for all the paths and all network loads.

In order to decide if we select $r=2$ or $r=3$, we have extended the analysis to other end-to-end paths and network loads (Table 3). Taking into account the combination of the CBR success rate and number of fit cases, we have observed that globally the differences between $r=2$ and 
$r=3$ are not significant. This leads us to set the best combination of $[k, r]$ parameters as $k=5$ and $r=2$ because slightly less computational load is required.

\begin{tabular}{|c|c|c|c|c|c|c|c|c|}
\hline \multirow[b]{2}{*}{$\begin{array}{c}\text { Mean } \\
\text { network } \\
\text { load }\end{array}$} & \multicolumn{4}{|c|}{$[k, r]=[5,2]$} & \multicolumn{4}{|c|}{$[k, r]=[5,3]$} \\
\hline & CBR\% & FitCases\% & \begin{tabular}{|c|} 
Ptx \\
mean savings \\
(dB)
\end{tabular} & \begin{tabular}{|c|} 
Ptx \\
mean savings \\
$(\%)$
\end{tabular} & CBR\% & FitCases\% & \begin{tabular}{|c|} 
Ptx \\
mean savings \\
(dB)
\end{tabular} & \begin{tabular}{|c|} 
Ptx \\
mean savings \\
$(\%)$ \\
\end{tabular} \\
\hline \multicolumn{9}{|c|}{ LP 1234} \\
\hline $30 \%$ & 97,86 & 99,82 & 1,3152 & 53,28 & 97,91 & 99,69 & 1,3234 & 54,21 \\
\hline $50 \%$ & 98,71 & 99,77 & 1,285 & 48,67 & 99,02 & 99,77 & 1,2892 & 49,57 \\
\hline $70 \%$ & 99,01 & 99,61 & 1,1832 & 43,69 & 99,19 & 99,73 & 1,17 & 42,8 \\
\hline $100 \%$ & 99,34 & 99,76 & 1,0654 & 37,3 & 99,46 & 99,71 & 1,0694 & 38,13 \\
\hline \multicolumn{9}{|c|}{ LP 34567} \\
\hline $30 \%$ & 94,39 & 98,98 & 1,386 & 40,44 & 94,46 & 98,81 & 1,434 & 42,06 \\
\hline $50 \%$ & 94,97 & 98,99 & 1,3632 & 37,67 & 95,34 & 98,86 & 1,3855 & 38,88 \\
\hline $70 \%$ & 95,91 & 98,69 & 1,2841 & 35,09 & 95,81 & 98,79 & 1,3027 & 35,71 \\
\hline $100 \%$ & 96,66 & 98,93 & 1,1703 & 31,48 & 96,87 & 98,64 & 1,1575 & 31,16 \\
\hline \multicolumn{9}{|c|}{ LP 456} \\
\hline $30 \%$ & 98,26 & 99,09 & 1,1534 & 33,77 & 98,05 & 99,19 & 1,1712 & 34,63 \\
\hline $50 \%$ & 98,47 & 99,26 & 0,9908 & 27,36 & 98,48 & 98,94 & 1,0039 & 28 \\
\hline $70 \%$ & 99,09 & 99,16 & 0,8974 & 23,66 & 98,98 & 99,12 & 0,8791 & 23,48 \\
\hline $100 \%$ & 99,35 & 99,21 & 0,8119 & 20,71 & 99,02 & 99,13 & 0,7876 & 20,07 \\
\hline \multicolumn{9}{|c|}{ LP 12} \\
\hline $30 \%$ & 99,92 & 99,45 & 1,0157 & 42,53 & 99,99 & 99,36 & 1,0548 & 44,61 \\
\hline $50 \%$ & 99,76 & 99,55 & 1,2148 & 44,43 & 99,7 & 99,42 & 1,2123 & 44,1 \\
\hline $70 \%$ & 99,8 & 99,1 & 1,2573 & 43,64 & 99,71 & 99,32 & 1,2566 & 43,63 \\
\hline $100 \%$ & 99,7 & 99,2 & 1,2983 & 43,67 & 99,56 & 99,2 & 1,3501 & 44,98 \\
\hline
\end{tabular}

Table 3. Evolution of overall results for $[k, r]=[5,2]$ and $[5,3]$ combination, for scheme MIN and mean network load evolution

As a conclusion, we can set for our work the optimum KB size as 300 previous cases and the CBR parameters as $[k, r]=[5,2]$.

Additionally, we have checked the best fitting $[k, r]$ parameters for CBR to ensure that every scheme was tuned for maximum performance in the final evaluation, thus avoiding a possible bias towards MIN. The results confirm that MIN is the best-performing scheme:

\begin{tabular}{|l|c|c|c|c|c|c|}
\hline Scheme & best $[\mathbf{k}, \mathbf{r}]$ & CBR\% & FitCases\% & CBR*FitCases(\%) & $\begin{array}{c}\text { Ptx } \\
\text { mean savings (dB) }\end{array}$ & $\begin{array}{c}\text { Ptx } \\
\text { mean savings (\%) }\end{array}$ \\
\hline MEAN & {$[7,1]$} & 99.89 & 88.45 & 88.36 & 0.3734 & 14.32 \\
\hline MIN & {$[5,2]$} & 98.71 & 99.77 & 98.48 & 1.285 & 48.67 \\
\hline FW & {$[1,1]$} & 99.82 & 81.98 & 81.83 & 0.4774 & 18.34 \\
\hline DW & {$[1,1]$} & 99.75 & 82.0 & 81.80 & 0.4561 & 17.27 \\
\hline
\end{tabular}

Table 4. Results for best [k,r] combination for LP 1234, all the schemes and $50 \%$ mean network load evolution

\subsection{Results}

Once we have fixed these work values, we have proceeded to analyze the global results regarding the success rate on the proposed transmission power for the exploitable cases and achieved power savings by the different scheme algorithms, under different network loads. Note that such a success rate comes from the CBR\% x FitCases\% product, namely, the \% of situation where $\mathrm{CBR}$ proposes a feasible solution actually improving the transmitted power. Figures 7 and 8 illustrate the obtained results. 


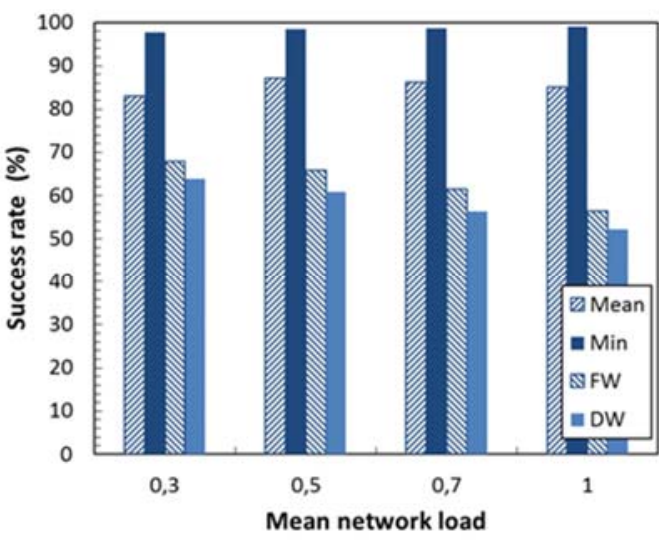

Figure 7. Evolution of CBR\% $x$ FitCases\% product as a function of the mean network load, for path LP 1234 and $[k, r]=[5,2]$

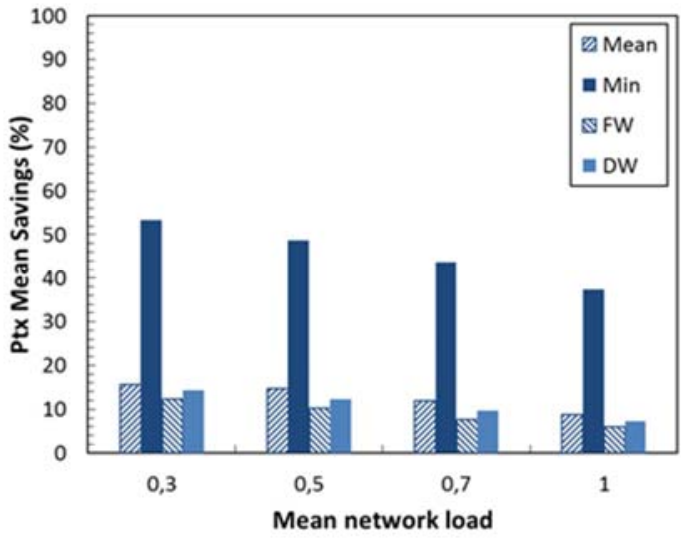

Figure 8. Evolution of Transmission Power mean saving with mean network load, for path LP 1234 and $[k, r]=[5,2]$

It can be observed in Figure 7 that for any network load, the best results regarding the success rate on the proposed transmission power exploitable for operational purposes are reached with the scheme MIN, which enforces the selection of our algorithm for the fine tuning of CBR parameters performed in the paragraphs above. The MEAN scheme could be the second option and our proposed FIXED-WEIGHT algorithm improves the success rate results compared to the DISTANCE-WEIGHT algorithm found in the literature [10]. Between these both options, FW presents better behavior by assigning static weights to past similar cases.

Regarding the mean savings in the transmission power (Figure 8), the MIN scheme provides clearly the best results for any mean network load. For example, the mean savings in the transmission power reaches around $49 \%$ for a mean network load of $50 \%$, and around $53 \%$ for a load of $30 \%$. It can be observed that the power savings are dependent on the network load; in particular, mean power savings are higher when the mean network load is lower. This can be explained because the conservative policies applied to set the System Margin are quite unnecessary when the traffic demand arriving to the network is not high. In terms of nominal power savings, for example, we have obtained a mean value of $1.285 \mathrm{~dB}$ (for $50 \%$ mean network load) and $1.3152 \mathrm{~dB}$ (30\% network load). This nominal power savings value corresponds to the global mean savings for the whole new incoming requests, including those when no power savings exist. And these results are similar for any of the four end-to-end paths considered, as shown in Table 2 for the MIN scheme.

These results show that the best option to propose an optimized transmission power is to apply the MIN scheme and $[k, r]=[5,2]$, with a success rate of $98 \%$ we can get $67 \%$ of exploitable operational cases, obtaining significant mean savings in the power to be transmitted to guarantee the end-to-end service, representing an advantage in the maintenance of the carrier operator. Moreover, this optimization is more significant for low network loads. 
In terms of different end-to-end paths, analysis of the results shown that in the success rate reached by CBR methodology there is a slight dependency on the length and number of links of the path; the best results are reached by using the MIN policy. When choosing this scheme, best results are globally obtained for medium length and short lightpaths, with the same behaviour shown for any network load, that is, success rate improves as mean load increases, even though results for the shortest path get decreased when load increases. Independently of the load, the best trade-off solution in CBR settings is to fix the k parameter to 5 , because even if a very slight CBR success rate reduction is observed, we obtained more volume of directly exploitable cases for operation and maintenance purposes, and this $k$ value does not lead to a hard computational load. We are indicating the best results, it does not mean that results are poor for the longest path with more links; on the contrary, for this end-to-end lightpath we obtain around $95 \%$ of CBR success rate and $99 \%$ of cases applicable to operation purposes under almost any network load. Regarding mean power savings, we have raised that there is a combined dependency on the length and the number of links of the path, the network load and the CBR settings; more nominal power savings are generally obtained for the paths composed of a larger number of links and this optimization is more significant when network load decreases, for any end-to-end lightpath, except for the shortest one, which shows the inverse trend. Again, we want to remark that results are also good for the shorter paths, with around $1.2 \mathrm{dBs}$, corresponding to more than $44 \%$ for any network load, for $\mathrm{k}=5$ parameter.

It is important to be noted that the power savings values are mean values. That is, in some cases, savings are considerably higher (e.g., reaching up to 3 and $4 \mathrm{~dB}$ ).

\section{CONCLUDING REMARKS}

In a general way, it has been observed that our approach to apply a cognition-based optimization strategy to set the transmission power allows getting significant power savings. Therefore, if adopted, it allows network maintenance costs reduction for carrier operators by reducing the operation margins. In particular, when considering typical paths, we have shown that by applying our cognitive proposal we can get success rates between $90 \%$ and $99 \%$ and power mean savings up to $1.31 \mathrm{~dB}$, representing a $53 \%$ improvement of the pre-assigned mean transmitted power. Optimized results either in the proposed transmission power and mean savings obtained are reached with the combination MIN scheme and $k=5$.

It has also been shown that, given a path, results depend on the network load: there is a certain dependency regarding the success rate and volume of fit cases reached for cognitive proposal. This dependency is much more significant in terms of power mean savings, achieving more efficiency in low load network conditions.

Globally the best results are obtained by selecting our MIN scheme proposal and even though it does not exist an optimized $[k, r]$ combination, valid for all the paths and all the network loads, we have observed that by setting CBR parameter $k$ to 5 , for most of the end-to-end lightpaths in any network load situation, the carrier operator can achieve a satisfying solution. Successful results are then obtained with CBR methodology for any end-to-end path. Based on a good trade-off between CBR success rate and power mean savings achieved, we can state that transmission power optimization leads to margins reduction and then our operational target is fulfilled, being more significant when network load is lower. 
This work opens several future research lines, as already pointed out in the paper. For example, additional performance benefits from adding new LP information in the KB online during the network operation (in addition -or alternatively- to the assumed offline $K B$ population and update with certain frequency) should be investigated, as well as the complexity/requirements that it would entail. Moreover, a complete architecture specification of the centralized SDN controller running CBR and able to control network nodes during the lightpath establishment and their quality assessment (CBR revise phase) would also be a key milestone toward the deployment of the paper proposals in production scenarios.

\section{REFERENCES}

[1]. Jean-Luc Augé, Can we use Flexible Transponders to Reduce Margins ?, OSA OFC/NFOEC Technical Digest, 2013.

[2]. J. Mitola III, G.Q. Maguire Jr., "Cognitive radio: making software radios more personal", IEEE Personal Communications, 6(4), 13-18, 1999.

[3]. G. S. Zervas and D. Simeounidou, "Cognitive Optical Networks: Need, requirements and architecture", in Proc. ICTON, 2010, paper We.C1.3.

[4]. T. Jiménez et al., "A Cognitive Quality of Transmission Estimator for Core Optical Networks", Journal of Lightwave Technology, 31(6), pp. 942-951, 2013.

[5]. Yvan Pointurier, Design of low-margin optical networks, Tu3F.5, OSA/OFC 2016.

[6]. T. Mitchell, Machine Learning, MacGraw-Hill, 1997.

[7]. A. Aamodt, E. Plaza, Case-Based Reasoning: Foundational Issues, Methodological variations, and System Approaches, AICOM - Artificial Intelligence Communications, IOS Press, Vol. 7: 1, pp. 39-59, 1994.

[8]. G.P. Agrawal Fiber-Optic Communication Systems, 3rd ed. John Wiley \& Sons, Inc, 2002.

[9]. J. M. Senior, Optical Fiber Communications, Principles and Practice, 3rd ed. Pearson Prentice Hall, 2009.

[10]. G. Keiser, Optical Fiber Communications, 3rd ed. McGraw-Hill, 2000.

[11]. A. Mitra, S. Kar, A. Lord, Effect of Link Margin on Spectrum Saving and Advantages of Flexigrid Optical Networking, IEEE, 2013 National Conference on Communications (NCC).

[12]. G. Keiser, FTTX Concepts and Applications, Wiley, 2006.

[13]. ITU-T Recommendation G.692 (10/98) - Optical interfaces for multichannel systems with optical amplifiers.

[14]. ITU-T Recommendation G.957 (03/2006) - Optical interfaces for equipments and systems relating to the synchronous digital hierarchy.

[15]. N. Fernández et al., "Virtual Topology Design and Reconfiguration using Cognition: Performance Evaluation in Case of Failure", V International Workshop on Reliable Networks Design and Modeling (RNDM 2013) co-located with ICUMT 2013, IEEE, 2013. 